\title{
Prediction of steel prices: A comparison between a conventional regression model and MSSA*
}

\author{
Martin Kapl and Werner G. MÜLleR ${ }^{\dagger}$
}

In this paper we compare two forecast procedures by its capabilities to predict market steel prices. One is based on the classical Box-Jenkins ARIMA class, the other on multichannel singular spectrum analysis (MSSA). We find the two approaches competitive with an advantage for MSSA to be expected as the time series grow longer.

Keywords AND PHRASEs: Hot rolled coil, Multi-channel singular spectrum analysis.

\section{INTRODUCTION}

Attempts to efficiently predict steel prices have generally been troubled by two issues: the qualification of which determinants should enter a specific model and whether a full market model with demand and supply equation could be produced. Less emphasis has been put on the choice of proper statistical prediction techniques, which is the focus of the present paper.

But let us first give a rough overview about the most relevant economic literature our study is based upon. Liebman in [26] examined the influence of safeguards on the steel price. Additionally to safeguards, he also chose the oil price, the industry production, the coal price, the scrap price, the dollar exchange rate, the industry salaries, the capacity of production and China's steel demand as regressors. In his findings, a cancellation of safeguards leads to an increase in the steel supply and as a consequence to a decrease of the steel price. The indicators for the Ukraine's steel price was analyzed by Yuzefovych in [31] through a simultaneous equation system. This equation system contained two single equations, one for the steel demand and another one for the steel supply. She derived that the steel demand is inelastic related to the price in what seems the only true market model considered in the literature. The structure of the steel market was found as a decisive factor for the steel price by Mancke [27]. He used the ratio of total imports and

*The findings of this paper are based on parts of the first author's PhD-thesis [25] at the Johannes-Kepler-University Linz, Austria. We are grateful to the generous support of his studies by voestalpine AG, Austria. Thanks go to Univ. Prof. Johann Brunner for sharing his economic expertise and to two anonymous referees for various useful remarks.

${ }^{\dagger}$ Corresponding author. total output (=import quota) as an instrument variable for the market structure. For him a simultaneous increase in the import quota and price is an evidence for perfect competition (a high domestic price influences the import quota positively). In contrast, an increase in the import quota and a decrease in the price indicate imperfect market conditions (the import quota is one factor which is responsible that the suppliers do not charge the full mark-up). All these papers have in common the aim to localize the determinants of the steel price. The industry production or the GDP, the exchange rate, the price of raw materials and the import quota can be named as the most important factors for steel price fluctuations. In none of these papers an attempt to forecast the steel price was made.

Since we needed a framework for our comparison of techniques a few premises had to be set:

- the prediction horizon should be at least 4 quarters (1 full year);

- it should be possible to estimate the variability of the predictions;

- quick rolling updates of the forecasts should be possible;

- and finally, we would ideally like to achieve some economic interpretability of our employed models.

Besides the classical ARIMA framework that was traditionally employed for purposes such as above, recently a new technique, the multi-channel singular spectrum analysis has emerged and proves to be competitive in various comparable circumstances, for a motivation see e.g. [19] and [22]. We will thus be employing these frameworks in the present paper.

\section{DATA}

Industrially produced steel comes in many shapes, thus we were required to focus on a specific product as the dependent variable in our forecast exercise, which turned out to be the so-called hot rolled coil. The time series for the respective prices in Germany, the USA and China are displayed in Figure 1. Eventually we are employing solely the German prices (solid line).

The set of determinant variables must be separated into those which effect the demand, and those effecting the supply. The first group contains the real GDP, the index of industrial production, the real effective exchange rate of the European Union with 12 main trade partners. On the other 


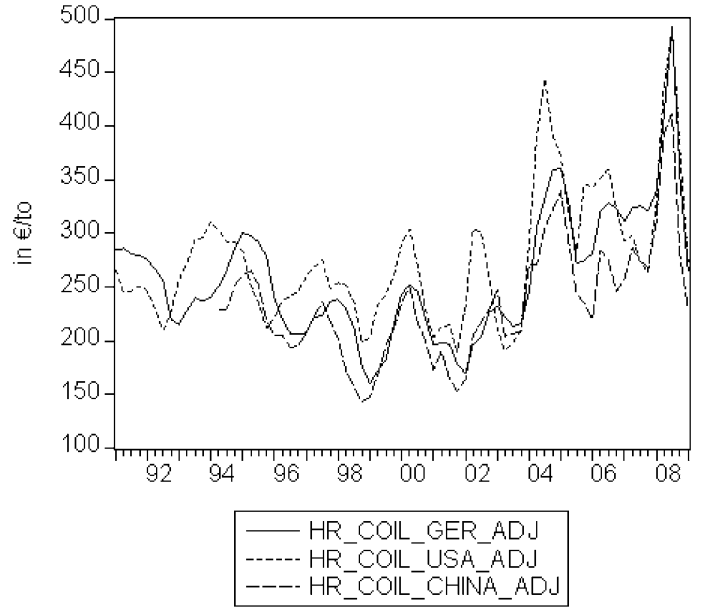

Figure 1. Hot-Rolled-Coil prices Germany, USA and China.

hand we have the prices of the raw materials of steel production, such as coke, scrap, oil and ore, which naturally negatively affect the supply.

Quarterly price data on hot rolled coil and raw materials were provided by the voestalpine AG. Macroeconomic data were drawn from the official Eurostat site [11], the index of raw materials comes from the Austrian National Banks data base [1]. The sample contains the period 1990Q1 until 2009Q1 except for some few missing values. In the following we also required some official growth rate predicitions, which were - together with the harmonized consumption price index - gathered from the homepage of the European commission [10]. All price data were adjusted for inflation by respective price indices and eventually properly back transformed (for details see [25]).

\section{MODELLING}

Due to the endogeneous character of price and quantity (causality in both directions) there are principally two possibilities for constructing viable regression based forecast models, i.e.

- a simultaneous equation system with a demand and supply function, or solely

- a price equation (reduced form), which includes all factors.

Although the latter does not allow for economic interpretation we were forced to use it, since contrary to [31] identification was not guaranteed. For the purpose of prediction, however, this was no essential drawback.

\subsection{Regression-based model}

For building classical ARIMA forecasts (see e.g. [5] and [16]) with covariates a thorough model search was undertaken. The regressors were chosen by a forward search started from a small initial model based on the Schwarz Information criterion. By the decision for unconditional forecasts and a forecast period of four quarters we were restricted to appropriately lagged factors. A couple of autoregressive structures were tested, although it eventually turned out that no autoregressive term was required. For the final specifiation formal diagnostic tests of the usual type (residual autocorrelation, stationarity, etc.) were undertaken.

It showed that a relatively simple linear non-autoregressive regression model containing a constant, predictions of the real GDP of the EU and the USA, the lagged hot-rolledcoil price of China and the USA, the lagged log of the coke price and the lags of the Dow-Jones index as well as the effective exchanges rates, which explained $80 \%$ of the insample variance, was the most preferable. We estimated it by 2SLS (see [30]) to accomodate errors in the predictor variables (cf. [16]), where all non-used regressors served as instruments. It will be used in the following exclusively (in [25] an $\mathrm{AR}(1)$ model with a different cofactor specification is compared with it) and will be referred to by ARIMA (indicating the model class within it was searched for). We do not disclose its concrete form, particularly its regression coefficients, for reasons of confidentiality. The estimation of this model yielded an in-sample $R^{2}=0.802$ with not unreasonable coefficient signs (i.e. negative for the European GDP, positive for the price of coke). Augmented Engle-Granger tests confirmed the cointegrated nature of the series.

\subsection{Multi-channel singular spectrum analysis (MSSA)}

We understand MSSA as a competitive non-parametric, data-adaptive technique for forecasting (longer) time series based on a separation of a set of series in their common interpretable components, like trends and cyclic oscillations and the non-interpretable component (noise). A standard (M)SSA consists of four steps:

1. Embedding

2. Singular value decomposition

3. Grouping

4. Diagonal averaging

as described in $[13,14]$ or elsewhere in this volume. We thus refrain from giving much more technical details on SSA and will emphasize aspects due to the multivariate character of our exercise.

Here a new trajectory matrix $\mathbf{X}$ will contain all lagged copies of all involved time series. Specifically for a given $S$-variate time series $Y=\left(Y^{(1)}, \ldots, Y^{(s)}, \ldots, Y^{(S)}\right)$, where $Y^{(s)}=\left(y_{n}^{(s)}\right)_{n=0}^{N-1}, s=1, \ldots, S$ and window length $L(1 \leq$ $L \leq N)$, the embedding-procedure will yield a sequence of $K=N-L+1$ lagged copies for each series and eventually a trajectory matrix:

$$
\mathbf{X}=\left[X^{(1)}, \ldots, X^{(s)}, \ldots, X^{(S)}\right]^{T}
$$


where

$$
X^{(s)}=\left(x_{i j}^{(s)}\right)_{i, j=1}^{L, K}=\left(\begin{array}{ccccc}
y_{0}^{(s)} & y_{1}^{(s)} & y_{2}^{(s)} & \ldots & y_{K-1}^{(s)} \\
y_{1}^{(s)} & y_{2}^{(s)} & y_{3}^{(s)} & \ldots & y_{K}^{(s)} \\
y_{2}^{(s)} & y_{3}^{(s)} & y_{4}^{(s)} & \ldots & y_{K+1}^{(s)} \\
\vdots & \vdots & \vdots & \ddots & \vdots \\
y_{L-1}^{(s)} & y_{L}^{(s)} & y_{L+1}^{(s)} & \ldots & y_{N-1}^{(s)}
\end{array}\right),
$$

$s=1, \ldots, S$, with the lagged vectors in its columns.

Of the matrix $\mathbf{X}$ we now require an eigentriple grouping in two disjunct parts, one corresponding to the signal, the other to the noise, for which we employ two methods. The first is based on the concept of weighted correlation or $w$ correlation introduced in [14, page 46f]. It is a measure for the dependence of two series defined by:

$$
\rho_{i j}^{(w)}=\frac{\left(Z^{(i)}, Z^{(j)}\right)_{w}}{\left\|Z^{(i)}\right\|_{w}\left\|Z^{(j)}\right\|_{w}}
$$

In the MSSA, $Z^{(i)}$ and $Z^{(j)}$ are stacked vectors of $S$-variate time series with individual length $N$, where

$$
\left(Z^{(i)}, Z^{(j)}\right)_{w}=\sum_{k=0}^{S N-1} w_{k} z_{k}^{(i)} z_{k}^{(j)},
$$

with $z_{k}^{(i)}$ and $z_{k}^{(j)}$ as k-th entry of $Z^{(i)}$ and $Z^{(j)}$, respectively, and $w_{k}$ as k-th entry of the weight vector $W=$ $\left(W^{(1)}, \ldots, W^{(s)}, \ldots, W^{(S)}\right) . W^{(s)}$ is given by $\left(w_{n}^{(s)}\right)_{n=0}^{N-1}$, $s=1, \ldots, S$, where

$$
w_{n}^{(s)}= \begin{cases}n+1 & \text { for } 0 \leq n \leq L^{*}-1 \\ L^{*} & \text { for } L^{*} \leq n<K^{*} \\ N-n & \text { for } K^{*} \leq n \leq N-1\end{cases}
$$

with $L^{*}=\min (L, K)$ and $K^{*}=\max (L, K)$ and

$$
\left\|Z^{(i)}\right\|_{w}=\sqrt{\left(Z^{(i)}, Z^{(i)}\right)_{w}}
$$

By using these equations one can achieve a full decomposition of the time series by constructing a $w$-correlation matrix of order $(d \times d)$ ( $d$ is the maximum number of eigentriples). Small absolute values of its entries $\rho_{i j}^{(w)}$ for $i \neq j=1, \ldots d$ indicate quasi $w$-orthogonality of the components $i$ and $j$, whereas large values stand for non-separability.

A graphical representation of this matrix (with grey shades for the correlation values) for the last forecast value by using all the time series relevant for our to be constructed MSSA model (see below) is given in Figure 2. One can clearly identify two orthogonal blocks, where the lower left can be attributed to the orthogonal components (the signal) and the upper right is indistinguishable from a white noise outcome.

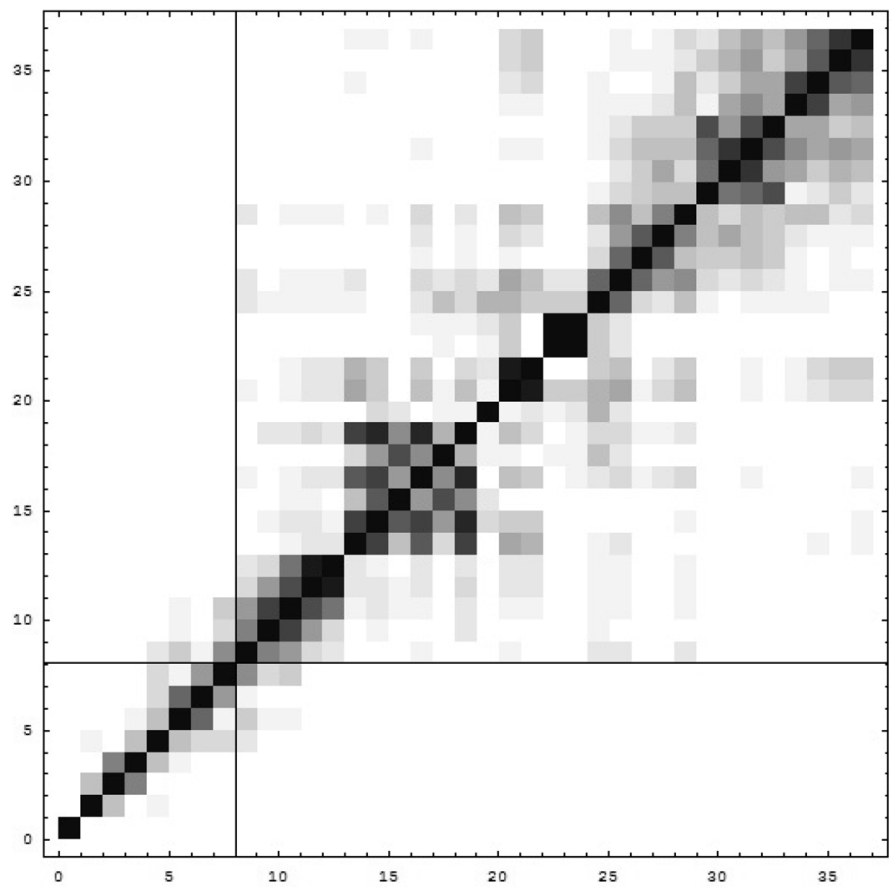

Figure 2. w-correlation matrix.

As a second method for the eigentriple separation we have generalized an algorithmic method developed for SSA by Vautard in [29]. An implementation was undertaken in Mathematica and the corresponding code can be found in [25, Appendix]. Here the optimal number of signal relevant eigentriples is calculated as follows:

- for a given order $p$ the eigentriple of the singular decomposition are separated into the sets $A=(1, \ldots, p)$ and $A^{\prime}=(p+1, \ldots, M)$, where $M$ is their maximum number;

- if the reconstructed part of the trajectory matrix $\mathbf{X}$ for a given set $A^{\prime}$ (start with $p=1$ ) is not dominated by white noise (evaluated by a specific Monte Carlo test procedure), then we increase $p$ accordingly by 1 and continue, otherwise the optimal order has been found.

Unfortunately it seems, that in the case of the multivariate generalizations, we tend to overdetermine the $p$, such that the power of the procedure is reduced. Therefore, although both methods have initially been used in our case study, for brevity, however, we will here report only results from the first.

After having determined a $p$ we can generate a forecast by using an approximate linear recurrent formula (the corresponding algorithm is described in [13, page 196]). The corresponding confidence bands were calculated by using an MSSA generalisation of the algorithm for generating bootstrap confidence intervals described in [20], for details see [25, page $44 \mathrm{f}]$. 
Eventually the final MSSA model was based on the following time series:

- hot rolled coil price for Germany and the USA;

- real GDP for the EU and the USA;

- index of the industrial production for the EU and the USA;

- the price of oil;

- the Dow Jones stock index.

The sample frequency was again quarterly and the data were deinflated by the HVPI which yielded 72 observations (1991Q2 until 2009Q1). A peridogram analysis (see [14, page 19]) showed a dominant trend for the German hot rolled coil price, for which case Golyandina et al. in [14, page 127] suggest to use a relatively large window length for the purpose of forecasting. Here, the largest possible corresponds to a choice of $L \approx$ observations $/ 2$; for the last forecast we thus chose $L=36$, for which a choice of $p=8$ determined by the first method described above turned out preferable. For other forecasts different values for $L$ and $p$ had to be used accordingly (for details see [25]).

\section{EVALUATION}

After the generation of point forecast the usually more delicate problem is evaluating its quality or the selection of the best forecast model among rivals. In this section we will discuss several evaluation methods and apply them to our models ARIMA and MSSA. For a recent discussion on the correct choice of evaluation criteria see [15].

\subsection{Recursive cross validation}

The perhaps most popular forecast model selection technique is to produce moving Out-Of-Sample (OOS) predicitons and is performed in the following way (cf. [5]):

1. Divide the complete series $(t=1, \ldots, T)$ into a training set from $t=1, \ldots, T^{*}$ and a validation set for evaluating the forecast performance from $t=T^{*}+1, \ldots, T$; set $L=T^{*} / 2$.

2. Estimate both models by using the training set and then produce OOS predictions (for 1 to 4 quarters) based on these estimates. Calculate the forecast error $\widehat{\epsilon}_{t+h, t}=y_{t+h}-\widehat{y}_{t+h, t}$.

3. Update the models with 1 additional observation $\left(T^{*}+\right.$ 1) and repeat above steps until the whole set is exhausted; update L.

4. At the end, calculate the root mean square error $\mathrm{RMSE}=\sqrt{\frac{1}{T-h-T^{*}} \sum_{t=T^{*}}^{T-h} \widehat{\epsilon}_{t+h, t}^{2}}$.

The results of the above procedure are displayed in Figure 3 and 4 and show the 1 to 4 quarter OOS predictions of the recursive cross validation together with a $95 \%$ bootstrap confidence band, the relative forecast error, the RMSE and the relative width of the confidence band.
The cross validation for the periods 2008Q3 until 2009Q1 also clearly demonstrates the limits of prediction models in cases of sudden structural breaks (financial crisis at the end of 2008). None of the tested models was able to capture the effects of the crisis, which lead to a significant increase of the relative prediction errors. Beside the deterioration of the predicition quality also the approximative confidence intervals proved too narrow, although they were eventually consistently increasing.

\subsection{Forecast optimality}

Another common evaluation criterion is defined by the following properties (cf. [5, chapter 12]):

- optimal forecasts are unbiased;

- they have white noise 1-step-ahead errors;

- their h-step-ahead errors are at worst MA $(h-1)$;

- the variance of the $h$-step-ahead errors is nondecreasing with h.

This optimality is usually tested by performing the socalled Mincer-Zarnowitz Regression (see [28] for a recent generalisation):

$$
y_{t+h}=\beta_{0}+\beta_{1} \widehat{y}_{t+h, t}+u_{t}
$$

If the predictions $\left(\widehat{y}_{t+h, t}\right)$ are optimal, one must expect that $\left(\beta_{0}, \beta_{1}\right)=(0,1)$ holds. Equation (1) is equivalent with

$$
\widehat{\epsilon}_{t+h, t}=\alpha_{0}+\alpha_{1} \widehat{y}_{t+h, t}+u_{t},
$$

an we would like to achieve parameter values of $\left(\alpha_{0}, \alpha_{1}\right)=$ $(0,0)$ for unforecastability, which is tested by a standard Wald-Test. For h-step forecasts we need to control for error autocorrelation. According to these tests, forecast optimality was validated for both ARIMA and MSSA, details can be found in [25, page $59 \mathrm{ff}]$.

\subsection{Direction of change}

Hassani in [21] introduced another measure, the so called direction of change (DC) criterion. It determines the proportion of correctly determined directions of the time series:

- Given the forecasts $\widehat{y}_{t+h, t}\left(t=T^{*}+1, \ldots, T\right), \widehat{z}_{t+h, t}$ takes the value of 1 if the direction is correctly identified, 0 else.

- $\widehat{z}_{T^{*}+1+h, T^{*}+1}, \widehat{z}_{T^{*}+2+h, T^{*}+2}, \ldots, \widehat{z}_{T+h, T} \sim$ iid. $\operatorname{Bernoulli}(p=1 / 2)$ with expectation $p$ and variance $p^{2}>0$.

- $S_{n}=\widehat{z}_{T^{*}+1+h, T^{*}+1}+\cdots+\widehat{z}_{T+h, T} \sim \operatorname{Binomial}(n, p)$, with $n=T-T^{*}$.

- $Z_{n}=\frac{S_{n}-n p}{p \sqrt{n}} \stackrel{D}{\rightarrow} \mathrm{N}(0,1)$ or $\frac{\sqrt{n}}{p}(\bar{z}-p) \stackrel{D}{\rightarrow} \mathrm{N}(0,1) ; \bar{z}=$ $S_{n} / n=\left(\widehat{z}_{T^{*}+1+h, T^{*}+1}+\cdots+\widehat{z}_{T+h, T}\right) / n$.

One thus yields $2 \sqrt{n}(\bar{z}-0.5) \sim N(0,1)$ as an asymptotic test statistic against the value 0.5 und thereby whether the directions can be identified significantly more often than by 

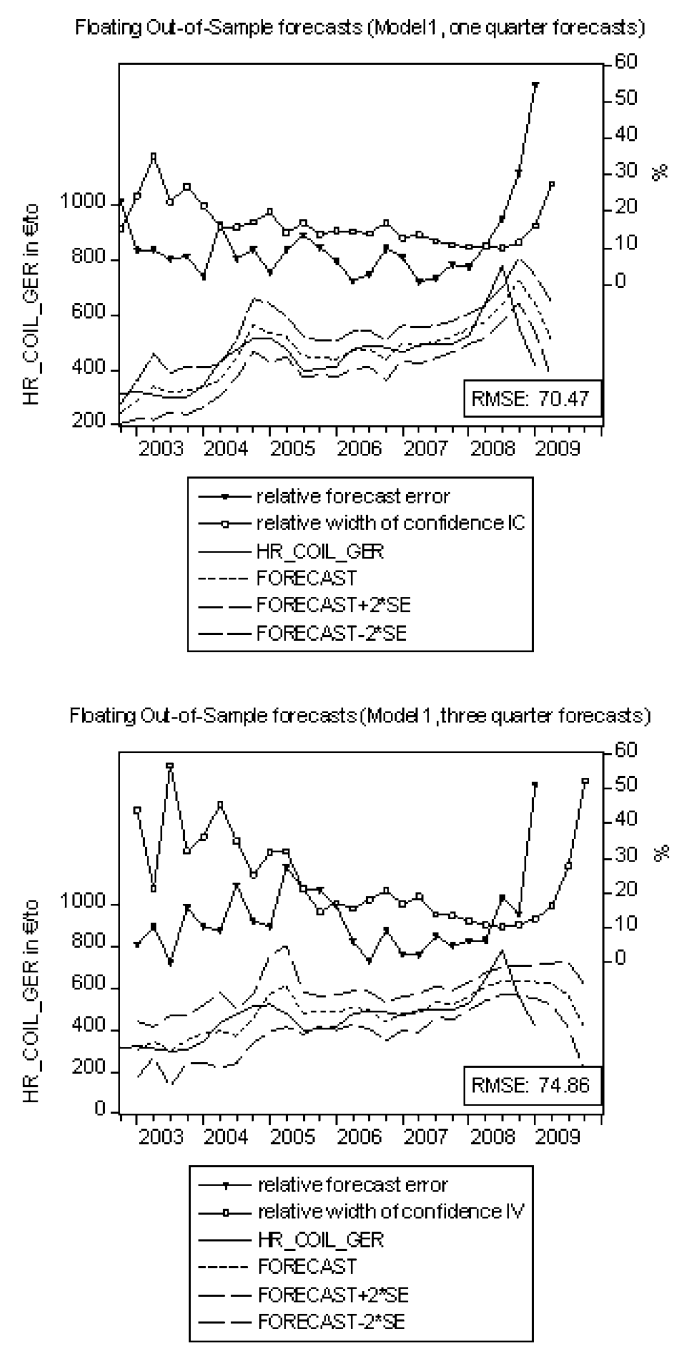
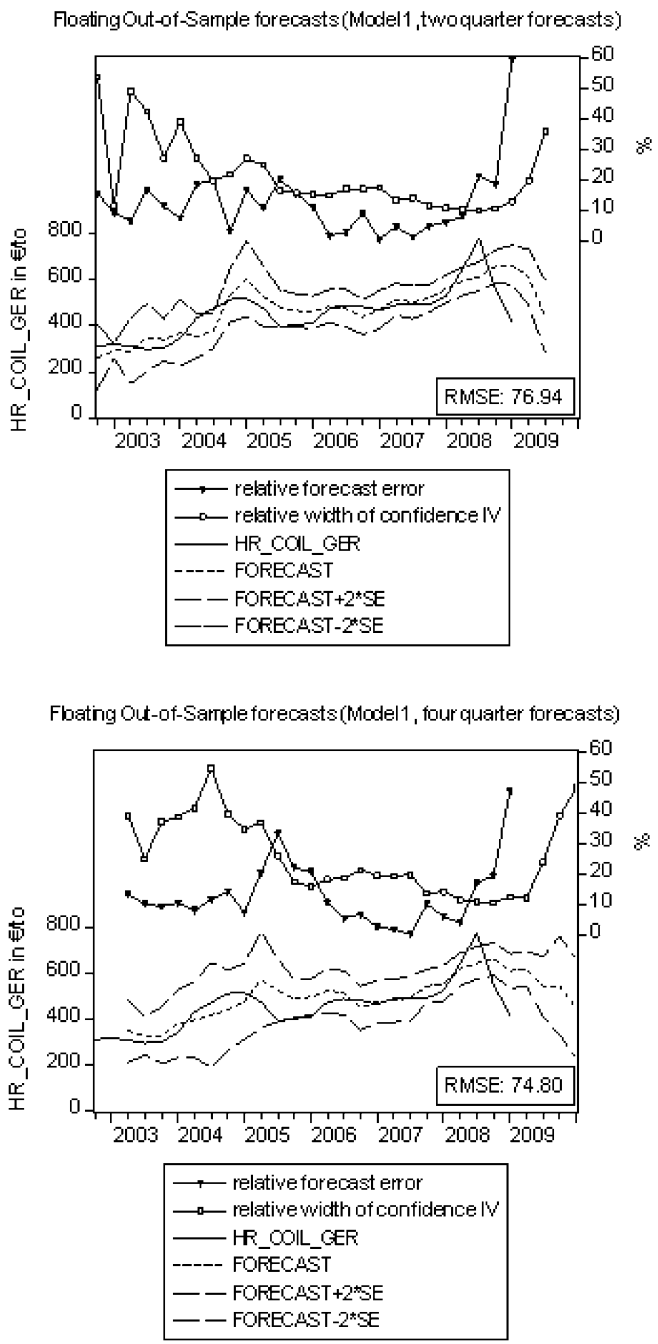

Figure 3. Predictions and recursive cross validation for ARIMA.

Table 1. Direction of Change

\begin{tabular}{|c|c|c|c|c|}
\hline \hline \multirow{2}{*}{ Model } & $h=4$ & $h=3$ & $h=2$ & $h=1$ \\
\cline { 2 - 5 } & $\bar{z}$ & $\bar{z}$ & $\bar{z}$ & $\bar{z}$ \\
\hline ARIMA & $0.83^{* * *}$ & $0.84^{* * *}$ & $0.80^{* * *}$ & $0.63^{*}$ \\
MSSA & 0.71 & $0.75^{*}$ & $0.77^{* *}$ & $0.9^{* * *}$ \\
\hline \multirow{2}{*}{ *** $1 \%$ significance, ${ }^{* *} 5 \%$ significance and ${ }^{*} 10 \%$ significance; }
\end{tabular}

pure random choice. The results for the two models is given in Table 1, which shows that the MSSA has the advantage for the shorter lags, whereas the regression model is slightly superior for the longer lags.

\subsection{Robustness}

Eventually we would like to check whether our employed models are robust in the sense

- that the estimated relationships amongst the variables are not arbitrary,
- and that thus the respective models are able to achieve stable predictions under artificially generated uncertainty.

For that purpose we simulate pseudo-data of the hot rolled coil price on 3 different levels of noise (i.e. $\sigma_{1}=10, \sigma_{2}=$ 20 and $\sigma_{3}=30$ ) by the following procedure (which is inspired by a similar technique in [23]):

1. By estimation of various models we yield the fitted values and residuals $\epsilon_{i}^{m}=y_{i}^{m}-\widehat{y}_{i}^{m}$ for $m=1,2, \ldots$.

2. Generating synthetic variables $y_{i}^{m *}=\widehat{y}_{i}^{m}+\epsilon_{i j}^{m}$, where $\epsilon_{i j}^{m}$ is generated from a parametrical bootstrap from a $N\left(0, \sigma_{j}\right)$-distribution with $j=1,2,3$.

3. Employ recursive cross-validation for estimating the models with the series $y_{i}^{m *}$ and calculate the respective RMSE.

4. Do that 100 times.

The results are given in Table 2 and show clearly that the MSSA based model is more robust for a higher level of 

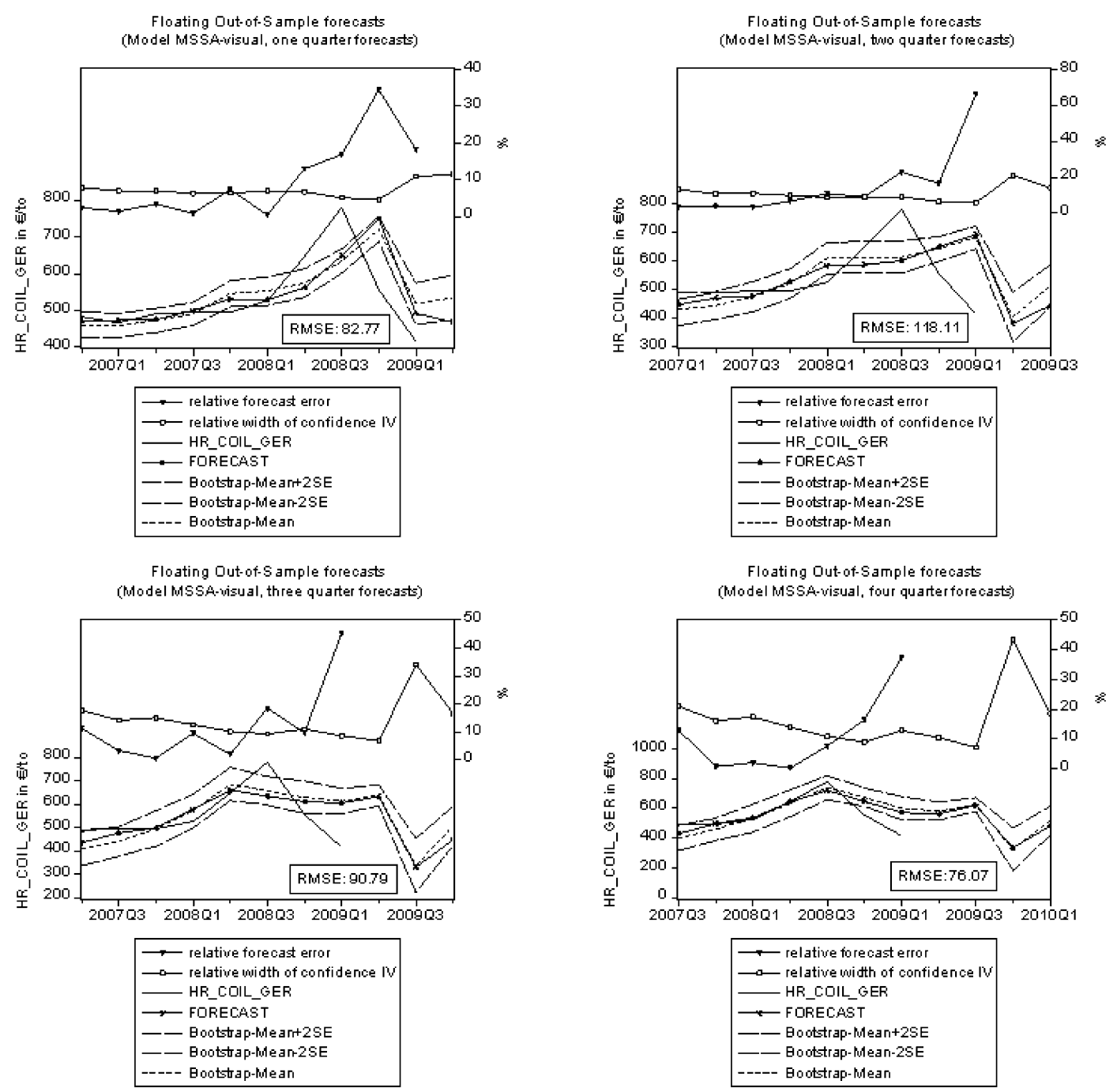

Figure 4. Predictions and recursive cross validation for MSSA.

Table 2. Robustness

\begin{tabular}{|c|c|c|c|}
\hline \hline Model & $\sigma=10$ & $\sigma=20$ & $\sigma=30$ \\
\hline ARIMA & $65.01(16.00)$ & $98.64(28.17)$ & $134.12(34.00)$ \\
MSSA & $81.23(9.75)$ & $89.59(15.31)$ & $107.17(27.78)$ \\
\hline
\end{tabular}

Average of the RMSE (standard deviations);

noise (the same holds for competition with other ARIMA models, which is described in [25]).

\section{CONCLUSIONS}

We have investigated the usefulness of MSSA for the prediction of steel prices as opposed to a conventional regression model from the ARIMA class. The following facts seem to emerge:

- the predictions are almost equivalent with respect to the RMSEs of the 4-quarter OOS;
- both models can be shown to be forecast optimal;

- the MSSA technique gives more robust models for higher noise levels;

- thus the prediction quality from both models is almost indistinguishable.

However, the main benefit of the MSSA seems to be the greater flexibilty for generalizations. In particular, we can, by recursive application of the described forecasting technique, construct predictions with a much larger forecast horizon than just four quarters. The addition of regressors without loss of degrees of freedom is another clear advantage. Furthermore it must be noted that MSSA makes no use of predicted values of regressor time series, which could be decisive, particularly when reliable forecasts for the regressors are not available.

Received 13 January 2010 


\section{REFERENCES}

[1] Austrian National Bank: http://www.oenb.at/

[2] Casella, G. and Berger, R. L. (2002). Statistical Inference, second ed. DUXBURY Thomson Learning.

[3] Clements, M. P. and Hendry, D. F. (2005). Evaluating a Model by Forecast Performance. Oxford Bulletin of Economics and Statistics 67 931-956.

[4] Davison, A. C. and Hinkley, D. V. (1997). Bootstrap Methods and their Application. Cambridge Series on Statistical and Probabilistic Mathematics. Cambridge University Press. MR1478673

[5] Diebold, F. X. (2007). Elements of Forecasting, fourth ed. Thomson South-Western.

[6] Efron, B. and Tibshirani, R. J. (1993). An Introduction to the Bootstrap. Monographs on Statistics and Applied Probability. Chapman \& Hall/CRC. MR1270903

[7] Elliot, G., Granger, C. W. J. and Timmermann, A. (2006). Handbook of Economic Forecasting. North-Holland.

[8] Elsner, J. B. and Tsonis, A. A. (1996). Singular Spectrum Analysis. A New Tool in Time Series Analysis. Plenum Press, New York.

[9] Engle, R. F. and Granger, C. W. J. (1991). Long-Run Economic Relationships: Readings in Cointegration. Advanced Texts in Econometrics. Oxford University Press.

[10] European Commission: http://ec.europa.eu/

[11] Eurostat: http://epp.eurostat.ec.europa.eu

[12] Ghil, M., Allen, M. R., Dettinger, M. D., Ide, K., Kondrashov, D., Mann, M. E., Robertson, A. W., Saunders, A., Tian, Y., VAradi, F. and Yiou, P. (2002). Advanced spectral methods for climatic time series. Reviews of Geophysics $\mathbf{4 0}(1)$ $1-41$.

[13] Golyandina, N. and Stepanov, D. (2005). SSA-based approaches to analysis and forecast of multidimensional time series. Proceedings of the Fifth Workshop on Simulation. 193-198.

[14] Golyandina, N., Nekrutin, V. and Zhigluavsky, A. A. (2001). Analysis of Time Series Structure: SSA and Related Techniques. Monographs on Statistics and Applied Probability 90. Chapman\&Hall/CRC. MR1823012

[15] Gneiting, T. (2009). Making and Evaluating Point Forecasts. arXiv.org:0912.0902.

[16] Greene, W. H. (2000). Econometric Analysis, fourth ed. Prentice-Hall, Inc.

[17] Grossman, G. H. (1986). Imports as a cause of injury: The case of the US steel industry. Journal of International Economics 20(3) 201-223.

[18] Gujarati, D. N. (2003). Basic Econometrics, fourth ed. McGrawHill.
[19] Hassani, H. (2007). Singular Spectrum Analysis: Methodology and Comparison. Journal of Data Science 5(2) 239-257.

[20] Hassani, H., Heravi, S. and Zhigluavsky, A. (2009). Forecasting European Industrial Production with Singular Spectrum Analysis. International Journal of Forecasting 25(1) 103-118.

[21] Hassani, H., Soofi, A. S. and Zhigluavsky, A. (2010). Predicting Daily Exchange Rate with Singular Spectrum Analysis. Nonlinear Analysis: Real World Applications in press (doi:10.1016/j.nonrwa.2009.05.008).

[22] Hassani, H. and Zhigljavsky, A. (2009). Singular Spectrum Analysis: Methodology and Application to Economics Data. Journal of Systems Science and Complexity (JSSC) 22(2) 372-395. MR2538869

[23] Jumah, A. and Kunst, R. M. (2008). Seasonal Prediction of European Cereal Prices: Good Forecasts Using Bad Models? Journal of Forecasting 27 391-406. MR2526235

[24] KaCAPyR, E. (1996). Economic Forecasting: The State of Art. M.E. Sharpe, New York.

[25] Kapl, M. (2009). Erstellung und Evaluierung von Prognosemodellen des Warmband-Stahlpreises. unpublished PhD-thesis, Johannes-Kepler-University Linz.

[26] Liebman, B. H. (2005). Safeguards, China, and the Price of Steel. Review of World Economics 142(2) 354-373.

[27] Mancke, R. (1968). The determinants of Steel Price in the US: 1947-65. The Journal of Industrial Economics 16(2) 147-160.

[28] Patton, A. J. and Timmermann, A. (2007). Testing Forecast Optimality Under Unkown Loss. Journal of the American Statistical Association 480 1172-1184. MR2412541

[29] Vautard, R., Yiou, P. and Ghil, M. (1992). Singular-spectrum analysis: A toolkit for short, noisy chaotic signals. Physica D $\mathbf{5 8}$ 95-126.

[30] Wooldridge, J. M. (2003). Introductory Econometrics, a Modern Approach, fourth ed. South Western, Mason.

[31] Yuzefovych, I. (2006). Ukrainian Industry in Transition: Steel Price Determination Model. Master thesis at the Kyiv School of Economics.

\section{Martin Kapl}

Johannes-Kepler-University Linz,

Austria

E-mail address: martin.kapl@gmx.net

Werner G. Müller

Johannes-Kepler-University Linz,

Austria

E-mail address: werner.mueller@jku.at 